\title{
ADVANCES IN LIPOSOMAL DRUG DELIVERY SYSTEM: FASCINATING TYPES AND POTENTIAL APPLICATIONS
}

\author{
SMITA BONDE, SUKANYA NAIR*
}

\author{
SVKM's NMIMs, School of Pharmacy and Technology Management, Shirpur Campus, Maharashtra, India
}

Email: smita.r@nmims.edu

Received: 18 Feb 2017, Revised and Accepted: 17 Apr 2017

\begin{abstract}
Liposomes are an efficient novel drug delivery system. They are used because of their structure which is stable and due to their ability to accommodate both lipophilic and hydrophilic drug. Various fascinating types of liposomes have been developed in recent past to further enhance their utility. Long-circulating liposomes or stealth liposomes are able to hide from the defence system of the body and circulate for a longer time in the blood. Targeted liposomes namely immuno- liposomes consists of antibodies conjugated on their surface to improve the specificity of the cell. Liposomes have been modified as per the conditions of $\mathrm{pH}$ and temperature, specifically designed to improve drug delivery to targeted tumor cells. Liposomes are being used in the treatment of various diseases and there are various liposomal drug formulations available today. Liposomes can be used as carriers for genetic materials such as antisense, DNA, RNA which are useful in the treatment of diseases. Liposomes are also efficient carriers of cytokines which further activate macrophages. This review provides the detailed insight of types and applications of liposomes and the potential challenges in the development of liposomal drug delivery systems.
\end{abstract}

Keywords: Targeted, Long-circulating, $\mathrm{pH}$ sensitive, Cancer, Antimicrobial agent

(C) 2017 The Authors. Published by Innovare Academic Sciences Pvt Ltd. This is an open access article under the CC BY license (http://creativecommons.org/licenses/by/4.0/) DOI: http://dx.doi.org/10.22159/ijap.2017v9i3.17984

\section{INTRODUCTION}

Liposomes are drug delivery systems which because of its organized structure can accommodate drugs depending on their solubility in aqueous as well as lipid phases. One of the features of liposomes is their ability to alter the biodistribution and pharmacokinetics of the drug associated. Liposomes are microscopic aggregates of highly ordered lipid molecules, dispersed in a hydrophilic solvent like water. Liposomes are amphiphilic having monolayer, one surface with polar head groups and other with fatty acid acyl chain. The monolayers are arranged in a lamella or a bilayer in which hydrophobic regions are sandwiched. This arrangement is similar to the phospholipids like phosphatidylcholines or lecithins which are the major component of the cell membrane. Liposomes take up the cylindrical shape and have a parallel arrangement but, a wedge shape is taken up by a liposome when there is a significant difference between the cross-sectional area of polar and non-polar regions. The differences may occur due to the electrostatic effects, arising because of the attractive or repulsive forces between adjacent head groups. In these conditions, curved structures are forcedly formed by amphiphiles, most common being the hexagonal phases (cylinders) and micelles (spheres to elongated rods). If the polar head group is broader than non-polar, the curvature will be positive and forms the outer surface of the curve. If the nonpolar tail is broader, the curvature will be negative with fatty acyl chains [1]. An extensive literature search was carried out to write this review. Some basic information on liposomes was collected from books. Most of the articles were collected from Science Direct, PubMed and Elsevier databases. Relevant articles were searched using keywords like liposomes, long circulating, $\mathrm{pH}$ sensitive, temperature sensitive, characterization, types of liposomes, applications of liposomes etc. Vast data was collected over the period of last 20 y to review the progressive developments in the field of liposome research.

\section{Morphology and nomenclature}

In dry state, the lamellar forming lipids exist in a dense crystalline form. The non-polar hydrocarbon chains are rigid and extend fully below a certain temperature and above this temperature, there is an increase in thermal excitation leading to distortion of chains. Above the transition temperature, the addition of water causes hydration of the polar heads of amphiphile and it rearranges into a lamellar form. There is diffusion of water through bilayer surface which causes a similar rearrangement of the lipid. The lipids arrange themselves into a series of parallel lamellae which by the addition of water is separated from the successor. When there is complete swelling of the liquid crystalline structure and equilibrium with excess water, closed packed multilamellar lipids are broken by mild agitation.

These liposomes which are large and possess a number of adjacent concentric bilayers and alternate layers of water are multilamellar vesicles (MLV). The interlamellar distance increases between adjacent lamellae due to charge-charge repulsive forces when ionic strength of hydration media is low. This occurs when lipid contains a negatively charged amphiphile component. Hydration, when occurs undisturbed with gentle stirring, individual lamellae detach to form large unilamellar vesicles (LUV). The maximum curvature that a lipid bilayer can achieve decides the lower limit of liposome size. Natural phospholipids such as egg and soy lecithins have a size of around $21 \mathrm{~nm}$. Small unilamellar vesicles (SUV) are single layered liposomes that have a size upper limit of around $100 \mathrm{~nm}$ [1].

\section{Types of liposomes}

\section{Long-circulating liposomes}

Stealth or long-circulating liposomes are sterically stable [2] and do not appear to the defence mechanism of the body mainly the Kupffer cells of liver and macrophages of the spleen [3]. The disadvantage of liposomes is the rapid clearance from the circulation, which can be avoided by using stealth liposomes [4]. It only takes minutes for intravenously administered drug to be engulfed by phagocytes and due to this, the drug does not reach the site of action. To increase circulation cohesive bilayers like disteroyl lecithin or sphingomyelin can be used [2]. The major reason for clearance is the adsorption of the blood macromolecules and their opsonization into the liposomal surface leading to phagocytosis [4].

Liposomes clear rapidly because of engulfment by the defence system or the disintegration due to plasma lipoprotein interaction. One of the reasons for the interaction with macromolecules is Van der Waals attraction. This hindrance can be resolved by sterical stabilisation. Sterical stabilization can be done by polyethylene glycol (PEG) modified phosphatidylethanolamine based lipid in the lipid preparation. Longer circulation is achieved by the liposome due to the dissociation of PEG-lipid from the layer which uses stronger hydrophobic anchors or polymerised bilayers. The electrostatic and hydrophobic interactions are prevented due to the high local 
concentration of hydrated groups at the surface of PEG-liposomes [2]. Stealth liposome named Doxil is present in the market as doxorubicin hydrochloride for treating AIDS-related Kaposi's sarcoma [4].

\section{Targeted liposomes}

Targeted liposomes include conjugating antibodies to the surface of the liposomes resulting in improved cell specificity. These are known as immuno- liposomes. On the surface of the liposomes, sulfhydryl groups of reduced immunoglobulin $\mathrm{M}$ (IgM) are conjugated to maleimide groups on the surface of liposomes. Maleimide groups are formed on the surface by liposome preparation using $\mathrm{N}-(\mathrm{m}-$ malemidobenzoyl): dipalmitoyl phosphatidyl ethanolamine (2.5 $\mu \mathrm{mol})$, dipalmitoylphosphatidylcholine $(25 \mu \mathrm{mol})$ and cholesterol (17.5 $\mu \mathrm{mol})$. Immunoliposomes show a specific cell type that corresponds to the antibody in vitro, but the cytotoxicity of the drug could not be retained by formulation [4]. The preparation of tumor specific liposomes is done by anti-BCG monoclonal antibodies coupling to $\mathrm{pH}$-sensitive liposomes. Modifications are done using succinylated polyglycidol (sucPG) to achieve binding specificity and internalization into cells by endocytosis. BCG-suc PG immuno- liposomes empty their contents into the cytoplasm by endosome fusion or fusion with liposomal membrane after target cell recognition and endocytic internalization [5]. The liposomal delivery to the liver parenchymal cell can be sustained and targeted by modifying distearoylphosphatidylcholine (DSPC) and cholesterol (Chol) liposomes in the ratio of 60:40 with a galactosylated cholesterol derivative (Gal-C4Chol) and tween 20 or 1, 2-distearoyl-sn-glycero-3-phosphoethanolamine-N-polyethylene glycol ( $\mathrm{PEG}_{\mathrm{x}}$-DSPE) when administered intravenously [6].

\section{Cationic liposomes}

When the DNA is mixed simply with cationic SUV, an effective lipidDNA complex is formed. This occurs due to a positive charge as a cationic lipid is used for neutralization of extended, polyanionic chain of nucleic acid and forms a compact microstructure lipoplex. Each particle possesses a positive charge which helps to approach and induce an interaction with the cell membrane which has a negative charge and by the receptor-mediated endocytosis they gain entry [1].

\section{pH sensitive liposomes}

pH-sensitive liposomes were first studied as an application which was designed to release their contents in a slightly decreased $\mathrm{pH}$ environment of 6 in a tumor tissue. They are used to improve the delivery of active substances to their site of action in the target cell. The components which at a physiological $\mathrm{pH}$ of 7.4 adopt a lamellar phase are used to form $\mathrm{pH}$-sensitive liposomes. The $\mathrm{pH}$ is decreased to a critical value around 5.5 due to which liposomes become fusogenic and fuse with the endosomal membrane. The fusion occurs due to the transition from a lamellar phase to a hexagonal phase which causes the lipophilic part of the lipid structure to have a larger space. The important criterion for the selection of the compound is that with a decrease in $\mathrm{pH}$ it must change its molecular shape [7]. The disadvantage of conventional liposomes includes degradation of the contents and liposomes during endocytosis, as the endosomal pH is reduced to 5 or less at the lysosome stage and also due to the enzymic action. When $\mathrm{pH}$ sensitive liposomes are used, the transition of non-bilayer takes place between early and late endosomal stages which upset the stability of endosome and the contents are delivered to the cytoplasm [1].

The major disadvantage of $\mathrm{pH}$-sensitive liposomes is its poor stability in vivo, due to which there is a leakage in the large liposomes prepared by dioleoylphosphaidylenthanolamine (DOPE) and oleic acid at $37^{\circ} \mathrm{C}$ in the presence of plasma and rapid clearance by the reticuloendothelial system. Leakage occurs due to the oleic acid loss into the plasma components. As a solution to the problem, liposomes with a double chain amphiphiles using 1, 2-dipalmitoylsn3 -succinylglycerol was prepared which was stable and even after prolonged plasma incubation maintained their $\mathrm{pH}$ sensitivity [1].

\section{Temperature sensitive liposomes}

For achieving the selective drug delivery action to tumor cells, temperature sensitive liposomes have been developed. Liposomes are prepared by the use of phospholipid mixtures having a transition temperature higher than body temperature by a few degrees. At temperatures near the transition temperature due to bilayer defects, the liposomes show increased permeability. When drugs containing cytotoxic drugs are injected into the systemic circulation and localized heat is applied externally to the target site, it causes the liposomes to release the drug to that area [1].

Modifying the liposomes with poly ( $\mathrm{N}$-isopropyl acrylamide) [poly (NIPAM)], results in the formation of temperature-sensitive liposomes. A hydrated coil to a dehydrated globule transition at $30^{\circ}$ $35^{\circ} \mathrm{C}$ in aqueous solution is exhibited by poly (NIPAM). The polymer is hydrophilic below the transition temperature but becomes hydrophobic above this temperature. Hence above the transition temperature, the liposomes are destabilized by polymer-liposome interaction. They are useful as a potential drug delivery system with temperature sensitivity, as the physiological temperature can be adjusted by copolymerization with hydrophilic comonomers [8].

\section{Applications}

\section{Cancer}

Undirected and ligand-targeted liposomes have been used to administer chemotherapeutic agents as well as peptides and proteins. Macrophages are activated and made tumoricidal by incorporating cytokines and other immunomodulators. The different anti-tumor effects are brought by using genetic materials like DNA, ribosomes, antisense molecules in liposomes. Most of these methods have given great outputs [1]

\section{Doxorubicin}

The drug resistance in cancer therapy is a big obstacle to successful cancer treatment. Drug resistance is caused by defective drug transport through the cell membrane when, drug inactivation is increased or drug activation is decreased, change in the affinity or levels of target enzymes and increased level of DNA damage repair. Doxorubicin is an anthracycline anticancer agent used for breast, leukemia, ovarian cancer treatment. Stealth monensin liposome (SML) is used to increase the potency of Doxorubicin. Liposomes are prepared using cholesterol, stearyl amine, dipalmitoyl phosphatidylcholine (DPPC), disteroyl glycerophosphoethanolamine coupled with polyethylene glycol (DSPE-PEG) and encapsulating radioactive monensin. Monensin increases the strength of Doxorubicin and acts as an immunotoxin against human tumors. In vivo circulation of liposomes is increased by using polyethene glycol (PEG) synthetic derivatives. The tests performed by preparing several formulations helped in estimating the amount of monensin entrapped, particle size, stability and leakage. The stearylamine used increased the formulation stability by producing a positive charge on the liposome surface which led to electrostatic repulsion between the adjacent bilayer. Disteroyl glycerophosphoethanolamine coupled with polyethylene glycol (DSPE-PEG) leads to steric hindrance and hence the entrapment by the reticuloendothelial system (RES) was reduced. Cholesterol used was responsible for increasing the stability and decreasing leakage. The stealth monensin liposomes containing monensin at a concentration of $10^{-9} \mathrm{M}$ was used which, is a non-toxic concentration. No Golgi dilation was observed. Depending upon the nature and difference in various human tumor cell lines, stealth monensin liposomes increase the activity of Doxorubicin by 2-2400 times [9]. Ehrlich tumor cells were inhibited using liposomes containing doxorubicin in the ratio of 100:100:60:4. The drug was delivered to the tumor cell by endocytosis. The results showed the presence of high concentration of doxorubicin in the tumor cells. Thus liposomal doxorubicin is more effective against Ehrlich tumor cells [10].

\section{Paclitaxel}

In our lab, we have studied the effect of the composition of phospholipids on drug entrapment efficiency and $\mathrm{pH}$ sensitivity. Paclitaxel-containing liposomes of different phospholipid compositions were formulated and compared. Liposomes with phosphatidylcholine alone did not show pH sensitivity. Another formulation with phosphatidylcholine/dioleylphodphatidylethanolamine (8:2) released drug at $\mathrm{pH} 5.5$, but was found to be 
unstable at $\mathrm{pH}$ 7.5. On inclusion of CHEMS, the liposomes were stabilized at physiological $\mathrm{pH}$, and released paclitaxel at a lower $\mathrm{pH}$. Thus the formulation composed of phosphatidylcholine/ dioleylphodphatidylethanolamine/cholesteryl hemisuccinate $(8: 2: 2)$ containing paclitaxel and lipids in the molar ratio of 1:30 (drug: lipid) has proved to be the most efficient $\mathrm{pH}$ sensitive system with $94 \%$ entrapment efficiency. This formulation showed $96 \%$ of drug release at pH 5 within 15 min [11]. It was further optimised by using Box-Behnken design. The aim was to investigate the combined influence of 3 independent variables in the preparation of paclitaxelcontaining $\mathrm{pH}^{-}$-sensitive liposomes. Independent variables selected were the molar ratio of phosphatidylcholine: ditolyl phosphatidylethanolamine (X1), the molar concentration of cholesteryl hemisuccinate (X2), and the amount of drug (X3). Liposomes were prepared by thin film hydration method and evaluated for the percent drug entrapment, vesicle size, and the $\mathrm{pH}$ sensitivity. The transformed values of the independent variables and the dependent variables were subjected to multiple regressions to establish a full model second order polynomial equation. $\mathrm{F}$ was calculated to confirm the omission of insignificant terms. The computer optimisation process and contour plots predicted the levels of independent variables for maximised response of percent drug entrapment with constraints on the vesicle size and the $\mathrm{pH}$ sensitivity [12].

\section{Peptides}

Peptides show the properties of tumor recognition, tissue penetration, selective binding and internalising capacity by the cancer cells. These properties make them useful for delivering oligonucleotides, toxins and radioactive molecules. Peptides are not detected by the immune system and there are less or no known side effects. Cell-specific targeting can be achieved by incorporating the peptides into multicomponent complexes. Peptides show excellent tumor cell penetration. The cell penetrating peptide (CPP) formulations were prepared for selective drug delivery and to increase the circulation time in vivo without affecting the cell penetration. These are smart stimuli-sensitive preparations whose functions can be turned on or off by outer stimuli-sensitive polyethene glycol (PEG). To penetrate the cytoplasmic membrane barrier for drug delivery, octa-arginines are utilised and to resist the kinetic barriers, a removable coating from cholesterol anchored reduction-sensitive PEG is used. For the studies, liposomes with functional lipids attached to different cholesterol were prepared. The composition used was egg phosphatidylcholine (EPC)/ cholesterol (chol) in the ratio of 65:35 and the cholesterol percentage decreased with the use of functional lipids attached to different cholesterol. The solvent used was chloroform which was evaporated using rotary evaporator [13].

\section{Docetaxel}

Docetaxel from taxane class of anticancer agents is a very important chemotherapeutic agent. Docetaxel induces a mitotic block at the metaphase or anaphase thereby inhibiting cell proliferation. Polymerization of stable microtubules is promoted, prevented from breaking apart and significantly affects the number of key cellular functions which depends on the turnover of tubulin.

Since docetaxel is known to prevent the depolymerization of microtubules, it is cytotoxic against human ovarian, endometrial, colon and breast cancer. When compared with insoluble paclitaxel, docetaxel which is soluble shows more cytotoxicity. Taxane liposomes are either conventional or PEGylated (polyethene glycol) are prepared by forcing out multilamellar liposomes by using different cholesterol ratios in the phospholipid mixtures. For evaluation of in vitro stability, cytotoxic activity, pharmacokinetics and biodistribution, the composition of liposomes are optimised to obtain maximum stability and incorporating docetaxel. The formulation in which liposome composition was egg yolk phosphatidylcholine (ePC), phosphatidylglycerol (PG), cholesterol (chol) i. e ePC/PG/chol in the ratio of 9:1:2 which contains encapsulated docetaxel proved effective in reaching a good drug concentration. The inclusion of small amount of cholesterol did not have any negative effect on docetaxel as compared to paclitaxel. However large amount affected the stability. Docetaxel shows better solubility and less rigidity as it is a semi-synthetic derivative of paclitaxel in which benzoyl group is replaced by a tertiary butyl ester group. This may minimise hydrophobic interactions between cholesterol and docetaxel in a lipid vesicle. Sterically stabilised liposomes containing docetaxel was prepared by PEGylated (polyethylene glycol) liposomes/cholesterol in the ratio of 9:1:2:0:7. Both conventional and PEG encapsulated docetaxel at the highest dose was stable at $4^{\circ} \mathrm{C}$ in the hydrated form. In fetal bovine serum at $37^{\circ} \mathrm{C}$ it was found to be unstable.

Liposomal docetaxel shows same in vitro cytotoxicity and better pharmacokinetic properties. When further compared with the docetaxel in tween 80 formulation it was found that liposome containing docetaxel showed an increase in plasma drug concentration and the drug ADME (absorption, distribution, metabolism and excretion) was increased up to $24 \mathrm{~h}^{*}$. In docetaxel encapsulated PEGylated liposome, there is a need to increase the concentration of drug within lipid bilayer without affecting liposome stability [14]

\section{Endelfosine}

Endelfosine is an anticancer agent which is an alkyl phospholipid analog. Endelfosine acts an anticancer agent by inducing cell apoptosis in tumor cell through a stress response from the endoplasmic reticulum and mitochondrial interaction. Endelfosine is known for dose-dependent rupture of red blood cells and toxic to bone marrow cell and other normal tissues. Therefore to remove side effects tumor-targeted delivery is adopted. The liposome surface is modified with AG73 peptides to improve delivery of endelfosine which is a lipophilic anticancer drug. The drug is delivered to the tumor cells which overexpress the syndecan 2 which is a cell surface receptor. To test the cellular uptake due to the surface density of liposomes modified with AG73 peptides, conjugated AG73 peptide polyethylene glycol with a molecular weight of 2000 was synthesized and encapsulated into anionic liposomes which where fluorescence dye-labeled with different ligand densities such as 1,2 , or $5 \mathrm{~mol} \%$ of total lipids. Increase in the cellular uptake of liposomes modified with AG73 peptides was proportional to the surface ligand density. For ligand mole percentages of 1,2 and 5 , the percentage cell positive for AG73 modified liposome was $19.8 \pm 2.0 \%, 23.1 \pm 5.0 \%$, and $99.2 \pm 1.0 \%$ respectively. The modified liposomes encased with endelfosine destroyed the cancer cells better than the unmodified liposomes. When tests were performed in tumor-bearing mice which, were administered intravenously with the AG73 peptide modified liposomes, exhibited greater accumulation of 2.1-fold in tumor cells as compared to unmodified liposomes [15].

\section{Methotrexate}

PEGylated methotrexate liposomes containing hydrogenated soy phosphatidylcholine and cholesterol have been prepared to achieve the targeted delivery to the tissues or organs that need it most. Attempts were made to enhance the encapsulation by use of nonionic surfactants such as tween-80, tween-20 and solubility enhancers such as $\beta$-cyclodextrin [16].

\section{Antimicrobial agents}

Bacterial, fungal, viral, parasitic diseases can be treated using liposomes by delivering the immunomodulators to the macrophages. The liposomes containing cytokines such an interferon $\gamma$ (INF- $\gamma$ ) which activates macrophages get the response of infectious diseases. Microorganisms that have undergone phagocytosis affects the host cell and start multiplication and shows resistance to treatment by antimicrobial drugs due to less penetration or when intracellular activity is reduced. The delivery of liposomes across the cell can be achieved by the phagocytic activity. It also protects the host from drug toxicity. Patients suffering from cancer, diabetes, AIDS have a high incidence of acquiring fatal systemic fungal infections and hence liposomes form an effective drug delivery system to treat such patients [1].

\section{Amphotericin B}

Amphotericin B is a polyene macrolide antibiotic used in the treatment of fungal infections of systemic nature, regardless of their 
toxicity. Molecular localization of amphotericin B entrapped in liposomes coated with polyethylene glycol (PEG) has been studied. The liposome composition included dipalmitoylphosphatidylcholine (DPPC), cholesterol (chol), distearoyl-N-(monomethoxy poly (ethylene glycol)succinyl) phosphatidylethanolamine-conjugated with polyethylene glycol (DSPE-PEG 2000) which was hydrated with $9 \%$ solution of sucrose and extruded. There was an increase in amphotericin B amount with the increased addition of DSPE-PEG while the amount decreased with the increase in cholesterol (chol). Methods like PEG/dextran two-phase partitions, potassium permeability measurement, circular dichroism (CD) spectroscopy and fluorescence quenching measurement were used to study molecular localization. According to the results, amphotericin B localization in PEG-liposomes are of two types, one which is the complex of amphotericin B with DSPE-PEG on the surface of membrane and other is amphotericin $B$ in a pore form in the hydrophobic core of liposome membrane. Amphotericin in both aggregated and monomeric state was present in PEG liposomes [17].

The effect of distearoylphosphatidylethanolamine conjugated with polyethylene glycol (DSPE-PEG ${ }_{1900}$ ) on toxicity and biodistribution of amphotericin B was studied in mice. Two formulations i.e. liposomes containing DSPE-PEG and amphotericin B (type 1 and type 2) and amphotericin B liposome without DSPE-PEG and also amphotericin B-deoxycholate (AMB-DOC) was compared for toxicity. The maximum tolerated a dose of AMB-DOC is $0.8 \mathrm{mg} / \mathrm{kg}$ per day which is shown in terms of death in the treatment of 5 consecutive days or increase in the monitoring parameters of renal and hepatic functions. The least toxic was amphotericin B liposome with the maximum tolerated dose $11 \mathrm{mg} / \mathrm{kg}$ per day. The DSPE-PEG containing amphotericin B liposome (type 1) was toxic similar to AMB-DOC. DSPE-PEG and amphotericin liposomes (type 2) had reduced toxicity with a maximum tolerated a dose of $9 \mathrm{mg} / \mathrm{kg}$ per day.

One comparing the biodistribution of DSPE-PEG and amphotericin B liposome (type 2) and amphotericin B liposome, it was found that biodistribution of DSPE-PEG and amphotericin liposomes (type 2) was increased as compared to amphotericin B liposome. In DSPEPEG and amphotericin B liposome (type 2), 30\% of the injected dose of amphotericin B was circulating in intact liposome whereas for amphotericin B liposome $6 \%$ was circulating at $24 \mathrm{~h}^{*}$ after administration [18].

\section{Leishmania infection}

Liposomes are an effective solution for the treatment of mononuclear phagocytes linked infectious diseases. Leishmaniasis is a fatal parasitic disease cause by protozoa leishmania spp which is an intramacrophage. The predominant drug used for treating leishmaniasis is antimonial even though they are toxic and their mechanism of action is unknown. When the anti-leishmanial agents are incorporated into the liposomes for delivery at the site of infection, they are more effective, have reduced toxicity and a few adverse effects. The study was carried out to develop novel liposomes containing meglumine antimoniate (MA), to study their effect on leishmaniasis and macrophageal uptake. 50\% inhibitory concentration $\left(\mathrm{IC}_{50}\right)$, when determined proved that MA containing liposomes were $\geq 10$-fold more effective with higher activity and increase in selectivity index by 5 -fold and decrease in toxicity by macrophages. When MA was incorporated in liposomes containing phosphatidylserine, the concentration required was $\geq 40$-fold lower to kill $100 \%$ intracellular amastigotes as compared to the free drug. Infected macrophages increased the uptake of fluorescent liposomes after a short incubation time as compared to the non-infected ones when studied through fluorescence microscopy. Thus the study proves that the use of MA incorporated liposomes is more effective for the treatment of leishmaniasis [19].

\section{Antisense}

Antisense are oligonucleotides (ONs) with a complimentary base sequence which are RNA specific. They prevent gene expression and are hence used in the treatment of cancer and inflammatory diseases. Oligonucleotides have poor solubility, weak cellular penetration and poor delivery to the cytoplasm. To solve this problem, anionic $\mathrm{pH}$ sensitive liposomes are prepared. Liposome endocytosis leads to the formation of intracellular vacuole i.e. endosome which undergoes fusion with lysosomes and inside of which the $\mathrm{pH}$ reduces due to a proton pump. The acidic $\mathrm{pH}$ destabilizes the $\mathrm{pH}$-sensitive liposomes and fuses with an endosomal membrane which leads to drug delivery in the cytoplasm [20].

Antisense oligonucleotides (asODNs) such as DNA or RNA, small interfering RNA i.e. siRNA and miRNA, DNAzymes, ribozymes which are oligonucleotides with catalytic activity are used to control gene expression [21]. Gene expression control by nucleic acids occurs by two mechanisms. Gene expression interference by antisense is done by preventing protein translation from mRNA. Short gene fragments of base sequence 15 to 20 are oligonucleotides (ONs) having a complementary sequence to target the mRNA. Hybridization of antisense oligonucleotides with mRNA occurs by base pairing by Watson and Crick which blocks protein translation and is known as translational arrest [19]. The second mechanism involves the activation of an enzyme RNase $\mathrm{H}$ which consumes the hybrid mRNA. ONs can also form a triple helix with double-stranded DNA and thereby control the gene expression or restrict transcription [21].

\section{SiRNA}

Small interfering RNA were first observed in plants in the 1980s, later in 1998 in Caenorhabditis elegans and further observed in mammals which led to their research [22]. They are capable of silencing post-transcription of all genes which are homologous and transgenes by incorporating long double-stranded RNA in various hosts.

The series of events that lead to the discovery of mRNA include, inside the cell the metabolism of double-stranded RNA (dsRNA) occurs leading to 21-23 interfering RNAs by the action of ribonuclease. Two RNA strands are unwound by a helicase activity when siRNA binds to RNA which induces silencing complex (RISC) and thus antisense binds to the focused RNA molecule. Hydrolysis of the target RNA at antigen binding site takes place due to endonuclease activity. Target RNA is destroyed when a single stranded RNA binds to the target RNA by Watson and Crick base pairing and activates the enzyme ribonuclease [23].

To improve solubility, distribution and circulation of oligonucleotides like siRNA and microRNA (miRNA), the liposomes are modified using polyethylene glycol (PEG). However, the long PEG chain reduces the efficient hybridization. The short PEG chain when used did not affect the gene silencing when studied in vitro [24]. The ability of antisense silencing of genes can be improved if liposomes are made of polyalkyleneoxide (poly (propylacrylic acid) varying in the grafting limit. The complexes with poly (propylacrylic acid) and polyalkyleneoxide, enhance the gene silencing in A20780 ovarian tumor cells [25].

\section{Herpes simplex virus-thymidine kinase}

Cancer cell targeting could be achieved through endothelial cells. Cationic liposomes carry a reporter gene i.e. lacZ; $\beta$-galactosidase. Sensitive liposomes are used to transfer a viral enzyme herpes simplex virus thymidine kinase (HSV-TK) to which ganciclovir binds and is phosphorylated. Ganciclovir triphosphate gets encapsulated into DNA that is elongated and acts as a pseudo base which leads to chain termination and cell apoptosis. The advantage is that, ganciclovir terminates the dividing cells and the liposome causes the DNA insertion that stays for a short time [26].

\section{Leukemia}

In leukemia there is an overexpression of the $\mathrm{BCl} 2$ gene and the development of cancer can be prevented if the $\mathrm{BCl} 2$ gene is reduced. Efficient liposomes have been prepared with good stability, specificity and toxic effects for the leukemia cells. The liposome formulation was composed of antisense oligonucleotides of which, the complex was formed with either cationic liquid, DOTAP $(1,2-$ dioleoyl-3-trimethylammoniumpropane), polyethyleneimine and polyethylene glycol modified liposome incorporation. The liposome surface is covered with a biomarker expressed on leukemia cell surface i.e. CD20, which are covalently bonded antibody recognizes. The expression of $\mathrm{BCl} 2$ gene is suppressed by the resulting immuno- 
liposomes. The polyplex or lipoplex liposome formulations combined with antibodies are efficient carriers for oligonucleotides (ONs) into human tumor cells [21].

The liposomes efficiently delivering drugs to the liver tissues are prepared by conjugation with $\mathrm{N}$-acetyl galactosamine. Physical properties such as particle size, specific ligand targeting when modified can be used to enable selective targeting for tumor cells. The barriers include endothelial, cellular membranes, endosome and drug accumulation in some other organ. Blood brain barrier of the brain has to be crossed for oligonucleotide therapy of nervous disorders. Instead of using $\mathrm{N}$-acetylgalactosamine (GAINAc), mellitin like polymer is used to attach the small interfering RNA (siRNA) which shows degradation in an acidic endosome environment. These additional PEG-masking ligand carriers called dynamic polyconjugate undergo a cleaving at an acidic $\mathrm{pH}$ and they release the cationic polymer charge which was masked previously. This method is designed to achieve the increased siRNA escape from endosome through an effect known as the proton sponge effect. The proton sponge effect occurs when; hydrogen ion influx is induced by water leading to endosome swelling due to which they disrupt [27].

\section{Vancomycin hydrochloride}

Vancomycin hydrochloride (VANH) is used in the treatment of osteomyelitis, is water soluble, glycopeptide antibiotic. It is used for the treatment of bacterial infections of the bone and the bone marrow, due to the contamination from injury, surgery or device fixation of orthopedic origin. VANH is incorporated into the liposomes to change a drug's bio-distribution, to improve therapeutic efficacy and reduce its toxicity. The liposomes were prepared with aim of gaining encapsulation efficiency (EE), a decrease in nephrotoxicity, increase circulation time in blood and efficient antimicrobial activity.

The liposome composition included soybean lecithin, cholesterol by the method of reverse phase evaporation. The experimental studies stated the optimum encapsulation efficiency was $40.78 \pm 2.56 \%$ and $188.4 \pm 2.77 \mathrm{~nm}$ was the mean particle size. VANH-liposomes showed sustained release characteristics when studied in vitro. The in vivo studies were carried out by administering the mice with VANHliposome intravenously which improved the mean resistance time (MRT) and AUC when compared to solution had increased significantly. The VANH accumulating in kidney was highly reduced by the VANH-liposomes when bio distribution studies were carried out intravenously and thereby reducing nephrotoxicity [28].

\section{Liposomal inhales}

The pulmonary route is a promising drug delivery route. The carriers used for this purpose are target selective and can control drug release. The advantages of the carrier system include decreased drug toxicity, increased the stability of the drug, and the local irritation is prevented. Nebulisers are used in the actual liposome formulation for inhalation. The nebulisers used include ultrasonic, air-jet and passively vibrating mesh nebulisers. The drug models with different molecular weights were used. Hydration method was used to prepare liposomes loaded with water soluble drug. Characterization of liposome was done by an extrusion method, which consisted of an extruder with $100 \mathrm{~nm}$ filter of polycarbonate. Various nebulisers were studied to find out which one would efficiently atomize the liposome preparation. Anderson cascade impactor (ACI) was used to measure the physical inhalation properties of liposomes. Through the studies, the most efficient nebulizer to deliver liposomal formulation to pulmonary parts was found to be air-jet nebulizer. The increase in liposome size caused leakage and decrease in lung delivery efficiency. Also the drugs with small molecular size showed leakage when atomized as compared to large molecular size. The leakage of small molecular size drugs could be decreased by decreasing particle size up to $100 \mathrm{~nm}$. The liposomal inhales could be prepared using air-jet nebulizer while taking into consideration the particle size [29].

\section{Epilepsy}

Gossypin liposomes have been prepared in a research study. They studied the efficacy of liposomal gossypin formulations by administering the formulation at two doses by the intravenous route. The studies were carried out in mice treated with pentylenetetrazole to induce seizures. They reported that the increased levels of malondialdehyde and glutathione were reduced and liposomal gossypin suppressed the progression of kindling in mice. These results suggest that liposomal gossypin appears to possess protective activity against kindling in mice [30].

\section{Acne vulgaris}

Liposomal gel of nadifloxacin have been prepared in order to achieve better permeation and high drug retention into the deeper layers of the skin for effective treatment of acne vulgaris [31].

\section{Liposome characterization}

Characterization of liposomes can be done in a number of ways which depends on the system aspects. These aspects show relation to the liposome structure, the number of bilayers or lamellarity, appearance and potential of the surface. Other relation is with liposome stability, which is concerned with the changes in vesicle size, storage, and vesicle ability to keep the entrapped drug intact in any medium like buffer or biological fluids. When unsaturated phospholipids containing liposomes are modified, the extent of modification must be determined during production or storage.

\section{Liposome structure}

Vesicle size can be estimated using the hydrodynamic methods like gel exclusion chromatography, or proton correlation spectroscopy, the value obtained is in terms of $\mathrm{z}$ average mean in nanometers or micrometers. The vesicle size distribution is given by polydispersity index. Surface charge on the vesicles are determined by the zeta potential (zP) and is useful in liposome formulations in which the drug to be encapsulated is charged which modifies the overall surface charge. The number of bilayers also termed as liposome lamellarity is estimated using small angle X-ray scattering which analyses whether the bilayers in the liposome are non-concentric like multilamellar vesicles. Structural or morphological studies are observed in varying conditions using light microscopy (large unilamellar vesicles and multilamellar vesicles) and electron microscopy. For samples in suspension, transmission electron microscopy is used which detects unilamellar, multilamellar and oligolamellar liposomes. Freeze-fracture electron microscopy (FFEM) is known to give a three-dimensional image of liposomes in suspension.

When spectroscopy is used for estimating turbidity of liposome suspension, calibration curves are used to estimate approximate vesicle size or concentration. The interaction of phospholipid polar heads with the counter ions and hydration can be estimated using Fourier transformation methods. Infrared or Raman spectroscopy can be used for estimating the dynamics and the structure of phospholipid acyl chains of the liposome, transition temperature can be estimated using nuclear magnetic resonance (NMR) and electron paramagnetic resource (EPR) [1].

\section{Light microscopy}

As restricted by the wavelength of light, a standard light microscope can resolve the structures that are $0.2 \mu \mathrm{m}$ and larger. A light microscope consists of a light source, condenser lens and an eyepiece. Modern microscopes are compound microscopes with more than one objective lens varying from $0.5-100$. Optically corrected immersion oil is used. There is image degradation due to the diffraction in a microscope having an objective magnification of 40X, 63X and 100X.3X to 1,500X. The image is viewed directly and can be recorded either by an attached video camera or by an attached camera photographically.

\section{Imaging modes}

Viewing the samples in a light microscopy is done using bright field imaging which uses optical density differences and a light colour that passes through the sample. The dark field is used for observing the small particulates and by background reversing and reversing the sample optical density increases the contrast. A phase contrast condenser can be utilized to increase the sample contrast when a phase shift between the transmitted and the scattered light passing 
through the sample is introduced. The three-dimensional images of light microscopy samples can be obtained by Hoffman modulation optics that uses a polarizer condenser. Analysis of polymers in a crystalline state is achieved by polarized light microscopy. Confocal light microscopes are special instruments that can penetrate thick samples and this is achieved when the plane of focus of the objective lens is controlled with stepper motors that are computer regulated [32].

\section{Transmission electron microscopy}

Transmission electron microscopy (TEM) is used to resolve structures having a small dimension less than $0.2 \mathrm{~nm}$ with the magnification range of less than 500 fold to million fold. TEM uses a heated filament that emits electron which is focused on obtaining images. TEM consists of lens in the form of electromagnets which bend the path of electrons. The electromagnets are also responsible for the electron acceleration and the energies used are in 60 to 100 $\mathrm{kV}$ range.

An aperture at the condenser limits the electron beam diameter which reaches the sample. There is an interaction between the atoms of the sample and the incident electron. There is a difference in the sample contrast due to the energy difference of electrons, occurring due to the atomic interactions. The aperture at the condenser controls the sample contrast. The objective lens focuses the beam of electron and the image is enlarged for observing through binoculars [32].

\section{Scanning electron microscopy}

The scanning electron microscopy (SEM) provides a threedimensional information of macro $(0.1-10 \mathrm{~mm})$, meso $(1-100 \mu \mathrm{m})$ and micro $(10-1000 \mathrm{~nm})$ structures in a micrograph. The particle size determination can be achieved using this technique. SEM gives a resolution of $3 \mathrm{~nm}$ and the range of magnification is less than 30 -fold to 300,000 fold. There is an acceleration of electrons emitted by a heated filament or when the lanthanum hexaboride crystal is excited at a voltage of 1-30 kV. After the electron is incident with the sample, a number of electrons are emitted with energy differences. The inelastic scattering from atoms at the sample surface leads to the formation of secondary electrons having low energy range of 0-50 $\mathrm{eV}$. The secondary electrons are collected by the detector which then gives a three-dimensional image.

The electrons due to the elastic scattering from the atom deep inside the surface are known as backscattered electrons and have high energy range. These are useful to distinguish between the atoms having a large difference in atomic number. The signals by the secondary, backscattered and X-ray (simple interaction with probe beam) are collected by the detectors, processed, amplified and displayed on the monitor [32].

\section{Photon correlation spectroscopy: determining particle size}

The photon correlation spectroscopy (PCS) measures the fluctuation intensity which is time dependent due to the Brownian motion of the particles in a scattered laser light. The mathematical process called correlation is carried out which measures the similarity between the signal and the separated signal by time decay. There is correlation decay due to randomness as the signals become out of phase with each other.

\section{Nuclear magnetic resonance}

By using nuclear magnetic resonance (NMR), the signal of phosphorus NMR of liposomes is measured before and after adding manganese ions in the mixture. Phosphorus in the outer surface of liposome interacts with the manganese ions which lead to the resonance signal broadening beyond detection. There are two peaks formed, one where the phosphorus interacts with manganese ions and other where there is no interaction as the phospholipid groups are within the vesicle and does not come in contact with the manganese ions.

The number of phospholipids in the outer layer can be estimated by comparison between the two signals. This technique exhibits the disadvantage that the negatively charged liposomes may undergo fusion due to the manganese ions causing a leak and interferes with the assay [33].

\section{Regulatory guidelines}

\section{Description and characterization}

The liposomal formulations should be characterized for position specificity, fatty acid composition, and the structural proof must be provided for well-defined synthetic or semisynthetic lipids, e. g. dimyristoyl phosphatidylcholine (DMPC). The lipid composition is studied in terms of percentage of lipid and fatty acid, the positional specificity of the acyl side chain, and the degree of fatty acid unsaturation.

\section{Lipid component manufacture}

Depending on whether the lipid utilized is naturally derived, synthetic or semi-synthetic information for manufacture must be provided.

\section{For synthetic and semi-synthetic lipids}

Complete information of the process and the purification procedure must be provided. The starting material specifications must be given for raw materials, reagents and solvents. If applicable, the controls for intermediates and critical steps which include controls that provide positional specificity of acyl chains must be mentioned.

\section{For naturally derived lipids}

The biological source should be given if the lipid is obtained from the animal source along with the information of the country of origin. It is required to provide information on the extraction and the purification procedures, as applicable. Information must be provided to ensure the removal, inactivation or avoidance of virus, animal proteins etc., wherever applicable. Appropriate manufacturing controls must be practised for avoidance or removal of pyrogenic material and bacterial endotoxins and the required description of the following must be provided.

Lipid component specifications: The useful lipid component must be distinguished from the lipids with similar structures by the identity test. The stability-indicating analytical procedure must be the basis of the assay. The validated analytical procedure with the information of validation must be provided. The information on impurity testing must be provided. For natural lipids, other tests to be provided are the degree of unsaturation of fatty acid side chain, the content of counter ion and divalent cation limits. For synthetic lipids, other tests that can be included are trans fatty acid, free fatty acid, peroxides, lysophospholipids, the content of counter ions and the divalent cation limits. It is required to provide information about the amount of impurity in final liposome drug product, structural alerts and the know toxicities of impurity. The impurities can be detected by comparing lipid under test with a reference standard using suitable analytical technique e. g. High-performance liquid chromatography (HPLC).

\section{Stability}

The stability studies must be conducted that includes stress testing at high and low temperature, the $\mathrm{pH}$ and oxygen to obtain storage conditions, the degradation profile determination and the appropriate stability-indicating analytical procedure development [34].

\section{CONCLUSION}

The researchers on liposomes have been going on since many years and are still prevalent. The aim of incorporating both hydrophilic and lipophilic drugs has been achieved through liposomes. Their structural stability and various modifications like $\mathrm{pH}$ sensitivity, temperature sensitivity, and sterical stabilization make them an efficient drug delivery system. The liposomes have proved to be a good drug delivery system for anti-cancer drugs and their applications have been increased significantly. Liposomes have also proved efficient in acting as drug delivery system for microbial infections in inhalation preparations. Target specific drug delivery is an important characteristic of liposomes. Liposomes must be formulated and characterised properly as per the intended use and the regulatory requirements. There is vast scope for research in 
liposomes to improve their chemical and physical stability and to develop the sterilisation methods to enhance their clinical utility.

\section{ACKNOWLEDGEMENT}

The authors would like to thank SVKMs NMIMS School of Pharmacy and Technology, Shirpur campus for the support and facilities provided for successful completion of the review article.

\section{CONFLICT OF INTERESTS}

Authors confirm that there are no conflicts of interest

\section{REFERENCES}

1. Kirby CJ, Gregoriadis G. Liposomes. In: Edith Mathiowitz. editor. Encyclopedia of Controlled Drug Delivery. Vol. 1. John Wiley and sons Inc., New York; 1999. p. 461-86.

2. Ceh B, Winterhalter M, Frederik PM, Vallner JJ, Lasic DD. Stealth ${ }^{\circledR}$ liposomes: from theory to product. Adv Drug Delivery Rev 1997;24:165-77.

3. Klibanov AL, Torchilin VP, Zalipsky S. Long-circulating sterically protected liposomes. In: Liposomes: a practical approach. $2^{\text {nded. }}$ New York: Oxford University Press Inc; 2003. p. 231-63.

4. Leach KJ. Cancer, drug delivery to treat local and systemic. In: Edith Mathiowitz. editor. Encyclopedia of Controlled Drug Delivery. Vol. 1. John Wiley and sons Inc., New York; 1999. p. 119-42.

5. Mizoue T, Horibe T, Maruyama K, Takizava T, Iwatsuru M, Kono $\mathrm{K}$, et al. Targetability and intracellular delivery of anti-BCG antibody-modified, $\mathrm{pH}$-sensitive fusogenic immuno- liposomes to tumour cells. Int J Pharm 2002;237:129-37.

6. Managit C, Kawakami S, Nishikawa M, Yamashita F, Hashida M Targeted and sustained drug delivery using PEGylated galactosylated liposomes. Int J Pharm 2003;226:77-84.

7. Süss RP, Schubert R. pH-sensitive liposomes. In: Weissig V, Torchillin VP. editors. Liposomes: a practical approach. $2^{\text {nd }}$ ed. New York: Oxford University Press Inc; 2003. p. 305-18.

8. Kono K, Henmi A, Yamashita H, Hayashi H, Takagishi T. Improvement of temperature-sensitivity of poly( $\mathrm{N}$-isopropylacrylamide)-modified liposomes. J Controlled Release 1999;59:63-75.

9. Fife K, Bower M, Cooper RG, Stewart L, Etheridge CJ, Coombes $\mathrm{RC}$, et al. Endothelial cell transfection with cationic liposomes and herpes simplex-thymidine kinase mediated killing. Gene Ther 1998;5:614-20.

10. Singh M, Ferdous AJ, Jackson TL. Stealth monensin liposomes as a potentiator of adriamycin in cancer treatment. J Controlled Release 1999;59:43-59.

11. Elbialy NS, Mady MM. Ehrlich tumor inhibition using doxorubicin containing liposomes. Saudi Pharm J 2015;23:182-7.

12. Rane S, Prabhakar B. Influence of liposome composition on paclitaxel entrapment and $\mathrm{pH}$ sensitivity of liposomes. Int J Pharm Tech Res 2009;1:914-7.

13. Rane S, Prabhakar B. Optimization of paclitaxel-containing $\mathrm{pH}$-sensitive liposomes by 3 factor, 3 level box-behnken design. Indian J Pharm Sci 2013;75:420-6.

14. Tang J, Zhang $\mathrm{L}, \mathrm{Fu} \mathrm{H}$, Kuang $\mathrm{Q}$, Gao $\mathrm{H}$, Zhang $\mathrm{Z}$ et al. A detachable coating of cholesterol-anchored PEG improves tumor targeting of cell-penetrating peptide-modified liposomes. Acta Pharm Sin B 2014;4:67-73.

15. Immordini ML, Brusa P, Arpicco S, Stella B, Dosio F, Cattel L. Preparation, characterization, cytotoxicity and pharmacokinetics of liposomes containing docetaxel. J Controlled Release 2003;91:417-29.

16. Shabnama, Srinivas P, Ravindra Babu DS. Formulation and evaluation of parenteral methotrexate nanoliposomes. Int J Pharm Pharm Sci 2014;6:295-300.

17. Shim G, Yu YH, Lee S, Kim J, Oh YK. Surface-modified liposomes for syndecan 2-targeted delivery of edelfosine. Asian J Pharm Sci 2016;II:596-602.
18. Moribe K, Maruyama K, Iwatshuru M. Molecular localization and state of amphotericin B in PEG liposomes. Int J Pharm 1999;193:97-106.

19. Van Etten EWM, Van Vianen W, Tijhuis RHG, Storm G, Woudenberg IAJMB. Sterically stabilized amphotericin Bliposomes" toxicity and biodistribution in mice. J Controlled Release 1995;37:123-9.

20. Borborema SET, Schwendener RA, Osso Junior JA, Andrade Junior HE, Nascimento N. Uptake and antileishmanial activity of meglumine antimoniate-containin liposomes in Leishmania (Leishmania) major-infected macrophages. Int J Antimicrob Agents 2011;38:341-7.

21. Fattal E, Couvreur P, Dubernet C. "Smart" delivery of antisense oligonucleotides by anionic pH-sensitive liposomes. Adv Drug Delivery Rev 2004;56:931-46.

22. Meissner JM, Toporkiewicz M, Czogalla A, Matusewicz L, Kuliczkowski K, Sikorski AF. Novel antisense therapeutics delivery systems: In vitro and in vivo studies of liposomes targeted with the anti-CD20 antibody. J Controlled Release 2015;220:515-28.

23. Ozcan G, Ozpolat B, Coleman RL, Sood AK, Berestein GL. Preclinical and clinical development of siRNA-based therapeutics. Adv Drug Delivery Rev 2015;87:108-19.

24. De Oliveira MC, Fattal E, Ropert C, Malvy C, Couvreur P. Delivery of antisense oligonucleotides by means of $\mathrm{pH}$ sensitive liposomes. J Controlled Release 1997;48:179-84.

25. Shokrzadeh N, Winkler KM, Dirin M, Winkler J. Oligonucleotides conjugated with short chemically defined polyethylene glycol chains are efficient antisense agents. Bioorg Med Chem Lett 2014;24:5758-61.

26. Peddada L, Garbuzenko OB, Devore DL, Minko T, Roth CM. Delivery of antisense oligonucleotides using poly(alkylene oxide)-poly(propylacrylic acid) graft copolymers in conjunction with cationic liposomes. J Controlled Release 2014;194:103-12.

27. Lorenzer C, Dirin M, Winkler AM, Baumann V, Winkler J. Going beyond the liver: progress and challenges of targeted delivery of siRNA therapeutics. J Controlled Release 2015;203:1-15.

28. Liu J, Wang Z, Li F, Gao J, Wang L, Huang G. Liposomes for systematic delivery of vancomycin hydrochloride to decrease nephrotoxicity: characterization and evaluation. Asian J Pharm Sci 2015;10:212-22.

29. Unida S, Ito $Y$, Onodera R, Tahara K, Takeuchi H. Inhalation properties of water-soluble drug-loaded liposomes atomized by nebulizer. Asian J Pharm Sci 2016;II:205-6.

30. Jacob JS. Characterization of delivery systems, microscopy. In: Edith Mathiowitz. editor. Encyclopaedia of controlled drug delivery. Vol. 1. John Wiley and sons Inc., New York; 1999. p. 234-49.

31. Nagpala D, Nidhi A, Katarec D. Evaluation of liposomal gossypin in animal models of epilepsy. Int J Pharm Pharm Sci 2016;8:247-51.

32. Shiny A, Toomu M, Dhurke RK. Enhanced dermal delivery of nadifloxacin using liposomes. Int J Appl Pharm 2016;8:53-9.

33. Zuidam NJ, Vrueh R, Crommelin DJA. Characterization of liposomes. In: Weissig V, Torchilin VP. editors. Liposomes: a practical approach. $2^{\text {nded. }}$. New York: Oxford University Press Inc; 2003. p. 31-76.

34. Liposome Drug Products. U. S. Department of Health and Human Services Food and Drug Administration Centre for Drug Evaluation and Research (CDER): Pharmaceutical Quality/CMC; 2015.

\section{How to cite this article}

- Smita Bonde, Sukanya Nair. Advances in liposomal drug delivery system: fascinating types and potential applications. Int J Appl Pharm 2017;9(3):1-7. 